\title{
Effects of Hypoxia on Oxidative Stress, Autophagy and Apoptosis in Cardiomyocytes
}

\author{
Qing-Min Feng1*, Yang Shao ${ }^{*}$, Rong Jiao ${ }^{2 *}$, Hong-Wei Wei ${ }^{*}$, Ming-Qiang Dai', \\ Hui-Xing Xie², Cai-Xia Xu², Ji-Ke Li" ${ }^{1 \#}$ \\ ${ }^{1}$ Cardiovascular Center of Hainan Medical University, The First Affiliated Hospital of Hainan Medical University, Haikou, China \\ ${ }^{2}$ Hainan Medical University, Haikou, China \\ Email: litf79997@aliyun.com, "jike0335@163.com
}

How to cite this paper: Feng, Q.-M., Shao, Y., Jiao, R., Wei, H.-W., Dai, M.-Q., Xie, H.-X., Xu, C.-X. and Li, J.-K. (2019) Effects of Hypoxia on Oxidative Stress, Autophagy and Apoptosis in Cardiomyocytes. Advances in Biological Chemistry, 9, 54-67. https://doi.org/10.4236/abc.2019.92005

Received: February 12, 2019

Accepted: April 21, 2019

Published: April 24, 2019

Copyright ( 2019 by author(s) and Scientific Research Publishing Inc. This work is licensed under the Creative Commons Attribution International License (CC BY 4.0).

http://creativecommons.org/licenses/by/4.0/ (c) (i) Open Access

\begin{abstract}
Coronary heart disease (CHD) is a hypoxia related disease. However, the relationship of the hypoxia-induced oxidative stress, autophagy and apoptosis in cardiomyocyte remains unclear. In this study, we used $\mathrm{CoCl}_{2}$ to mimic hypoxic conditions in $\mathrm{H} 9 \mathrm{c} 2$ cardiomyocytes and study the effects of $\mathrm{CoCl}_{2}$-induced hypoxia on oxidative stress, apoptosis and autophagy, as well as the relationships among these processes. Cell viability and levels of ROS, LC3-II, p62, caspase-3 and PARP were assessed. The viability and morphology of cardiomyocytes were affected by hypoxia, and hypoxia enhanced levels of ROS and the levels of the LC3-II, p62, caspase-3 and PARP proteins in H9c2 cells in a dose-dependent manner. ROS levels rise gradually in the presence of hypoxia; however, it shrinks when hypoxia reaches a certain level. Caspase- 3 and PARP levels were raised with the increasing of hypoxia level. Enhanced level of LC3 and decreased levels of p62 in hypoxic cells indicate that autophagy levels are in accord with hypoxia. Based on these results, hypoxia induces oxidative stress, apoptosis and autophagy in cardiomyocytes. Autophagy is a double-edged sword. At a low level, autophagy can resist oxidative stress and protect cardiomyocytes from oxidative stress, while high level autophagy can promote apoptosis of cardiomyocytes.
\end{abstract}

\section{Keywords}

Hypoxia, Oxidative Stress, Autophagy, Apoptosis, Cardiomyocyte

\section{Introduction}

Coronary heart disease (CHD) is the leading cause of death in older men and women in the western world, and reduced oxygen availability may cause or ex-

${ }^{*}$ Contributed equally. 
acerbate symptoms of CHD [1]. A constant oxygen supply is essential for cardiac viability and function. Hypoxia induces some pathological process, such as oxidative stress, autophagy and apoptosis [2] [3] [4]. To date, researchers have obtained a good understanding of individual pathological processes induced by hypoxia. However, little information is known about the relationships among these processes in cardiomyocytes which limited our understanding of the roles of oxidative stress, autophagy and apoptosis in the progression of human diseases [5].

Oxidative stress is defined as an imbalance between the reactive oxygen species (ROS) scavenging and producing systems in the organism [6]. ROS are implicated in irreversible damage to the cell membrane, DNA, and other cellular structures by oxidizing lipids, proteins, and nucleic acids [7]. However, ROS are markers of oxidative stress that are increased under pathological condition [8]. ROS levels are assessed using 2,7-dichlorodihydrofluorescein diacetate (DCFH-DA). Hypoxia inducible factor (HIF) is activated by hypoxia and is a master regulator of oxygen homeostasis, as it regulates the expression of many genes [9]. The expression and activity of the HIF-1 $\alpha$ subunit are regulated by cellular oxygen levels, and hypoxia increases HIF-1 $\alpha$ levels by inhibiting proline hydroxylation and the subsequent degradation of HIF-1 $\alpha$ [9].

Autophagy, an evolutionally conserved process of controlled cellular cannibalization, plays a vital role in cardiac physiology. Perturbations in cardiomyocyte autophagy contribute to the pathogenesis of a wide range of cardiac diseases, many of which culminate in heart failure [10]. LC3 is the only known protein that is specifically associated with all types of autophagic membranes, including phagophores, autophagosomes and autolysosomes [10]. Therefore, the amount of LC3-II correlates well with the number of autophagosomes, which provides a good index of autophagy induction [9]. Levels of p62 have also been used to monitor autophagic flux, and p62 serves as an adaptor protein that links ubiquitinated proteins to the autophagy machinery and facilitates their clearance in the lysosome [11]. Notably, p62 and p62-bound ubiquitinated proteins become incorporated into the completed autophagosomes and are degraded in autolysosomes [12]. Since p62 itself is mainly removed by autophagy, its expression is generally considered to inversely correlate with autophagy activity [12]. The accumulation of p62 has been used as a marker of suppressed autophagy, and similarly, a decrease in the p62 level indicates autophagy activation [11].

Apoptosis is the process of programmed cell death that occurs during heart development [13]. Caspase-3 is a common effector of most apoptotic pathways and is able to cleave several target proteins whose degradation will contribute to the execution phase of the cell death program [14]. Poly ADP-ribose polymerase (PARP) is a conserved nuclear enzyme that is present throughout the phylogenetic spectrum. The precise physiologic role of PARP is to induce DNA repair and maintain genomic integrity [15]. PARP has a complex role in cells induced by DNA damage, and PARP cleavage by caspases is a marker of apoptosis [15].

In patients with $\mathrm{CHD}$, the most effective therapy to reduce ischemic myocar- 
dial injury and infarct size is efficient myocardial reperfusion and early sustained restoration of blood flow (reperfusion) through the occluded coronary artery [16]. However, the myocardial reperfusion process also induces further myocardial cell death, a phenomenon known as myocardial ischemia/reperfusion (I/R) injury [17]. Diverse pathological process, such as oxidative stress, autophagy and apoptosis, contribute to the pathological mechanism underlying CHD [16], So far, researches mainly focus on the single pathological process caused by hypoxia, and there are few studies on the relationship among the three pathological processes [18].

In our study, we used $\mathrm{CoCl}_{2}$ to mimic hypoxic conditions in cardiac $\mathrm{H} 9 \mathrm{C} 2$ cells [19] and observed hypoxia-induced oxidative stress, autophagy and apoptosis. We examined the relationships among oxidative stress, apoptosis and autophagy. We believe that an understanding of oxidative stress, autophagy and apoptosis in hypoxic cardiomyocytes and exploring the relationship among the three are important for better clarifying the mechanism of $\mathrm{CHD}$ and should have important clinical significance in the prevention and treatment of this disease.

\section{Materials and Methods}

\subsection{Cell Culture}

The cardiomyocyte cell line H9C2 was obtained from the American Type Culture Collection (ATCC; Manassas, VA, USA). Cells were cultured in DMEM containing $10 \%$ fetal bovine serum (FBS) and a $1 \%$ antibiotic-antimycotic solution (100 U/ml penicillin and $100 \mu \mathrm{g} / \mathrm{ml}$ streptomycin). Cells were maintained at $37^{\circ} \mathrm{C}$ in a humidified atmosphere containing $5 \% \mathrm{CO}_{2}[20]$.

\subsection{Hypoxia Induction}

The hypoxia model was established using the hypoxia-inducing agent $\mathrm{CoCl}_{2}$, as described in a previous study [20]. Cells were plated in 96-well microtiter plates at a density of $1 \times 10^{5}$ cells $/ \mathrm{ml}$ and cultured with DMEM supplemented with $10 \%$ FBS for $6 \mathrm{~h}$ at $37^{\circ} \mathrm{C}$. Cells were subsequently incubated in serum-free $\mathrm{CO}_{2}$-independent DMEM supplemented with various concentrations of $\mathrm{CoCl}_{2}$ (300, 600, 900 or 1200 $\mu \mathrm{M})$ for $16 \mathrm{~h}$. Normoxic control cells were incubated under the same conditions in a normal atmosphere. Normoxic cells received normal serum and no $\mathrm{CoCl}_{2}$ treatment, and the hypoxic cells were then incubated in a hypoxic chamber.

\subsection{Cell Viability Assessment under Various Hypoxic Conditions}

MTT was added at a final concentration of $25 \mathrm{mg} / \mathrm{ml}$. After a 4-h incubation at $37^{\circ} \mathrm{C}$, the reaction was halted by adding $150 \mu \mathrm{l}$ of dimethyl sulfoxide (DMSO), and the relative absorbance value (AV) was measured at $595 \mathrm{~nm}$ using a microplate reader [21]. Cell viability was calculated according to the AV and density of cells.

\subsection{Western Blot Analysis}

Cells were lysed with radioimmunoprecipitation assay buffer [50 mM Tris- $\mathrm{HCl}$ 
( $\mathrm{pH} 7.4), 150 \mathrm{mM} \mathrm{NaCl}, 0.1 \%$ SDS, $1 \% \mathrm{NP}-40$, and protease inhibitor cocktail] for $30 \mathrm{~min}$ and centrifuged at $12,000 \times \mathrm{g}$ for $10 \mathrm{~min}$ prior to supernatant collection. The protein concentration was quantified using the bicinchoninic acid assay. Equal amounts $(60 \mu \mathrm{g})$ of protein were loaded into each lane of $10 \%$ SDS-PAGE gels, separated, and then transferred to polyvinylidene fluoride (PVDF) membranes (Roche Diagnostics $\mathrm{GmbH}$, Mannheim, Germany). After blocking with 5\% fat-free milk in Tris-buffered saline-Tween-20 (TBST) for $1 \mathrm{~h}$ at room temperature, PVDF membranes were incubated with primary antibodies (1:2000) overnight at $4^{\circ} \mathrm{C}$. Membranes were washed with TBST, incubated with horseradish peroxidase-conjugated anti-rabbit (L3012, Signal way Antibody, Nanjing, China) or anti-mouse (L3032, Signal way Antibody) secondary antibodies in TBST (1:5000) for $1 \mathrm{~h}$ at room temperature, and then visualized using a super enhanced chemiluminescence detection reagent (Beyotime Institute of Biotechnology, Haimen, China). Signals were detected using an Image Station 4000 R (Kodak, Rochester, NY, USA). Results were quantified using ImageJ software version 1.44 (National Institutes of Health, Bethesda, MD, USA). Each experiment was repeated separately at least three times.

\subsection{Fluorescence Assay of Intracellular ROS Levels}

Intracellular ROS generation was determined using the ROS-dependent oxidative conversion of cell-permeable DCFH-DA dye to the fluorescent dye dichlorofluorescein. H9C2 cells were treated as described above, washed 3 times with PBS, and incubated with a $10 \mu \mathrm{M}$ DCFH-DA solution in serum-free medium at $37^{\circ} \mathrm{C}$ for $30 \mathrm{~min}$ in the dark. Cells were washed 3 times with PBS, and ROS concentrations were quantified using a ZEISS LSM 510 confocal microscope at a wavelength of $488 \mathrm{~nm}$.

\subsection{Statistical Analysis}

All data are presented as means \pm SD. Data were analyzed using SPSS software version 19.0. Statistically significant differences were determined using one-way analysis of variance (ANOVA) with the LSD post hoc test was performed. Notably, $P<0.05$ was considered to indicate a statistically significant difference [21] [22].

\section{Results}

\subsection{Effects of Hypoxia on the Viability of H9C2 Cells}

Based on the results from the MTT assay, the $\mathrm{CoCl}_{2}$ treatment decreased cell viability in a concentration-dependent manner (Figure 1). Cell viability decreased after a $16 \mathrm{~h}$ incubation with increasing concentrations of $\mathrm{CoCl}_{2}(300$, $600,900$, or $1200 \mu \mathrm{M})$ compared with the control group $(P<0.05)$, and $600 \mu \mathrm{M}$ was sufficient to decrease viability by $\sim 50 \%$. At a concentration of $300 \mu \mathrm{M}$, $\mathrm{CoCl}_{2}$ began to exert cytotoxic effects, and cell viability gradually decreased as the $\mathrm{CoCl}_{2}$ concentration increased, reaching a minimum at $1200 \mu \mathrm{M}(P<0.05)$. 


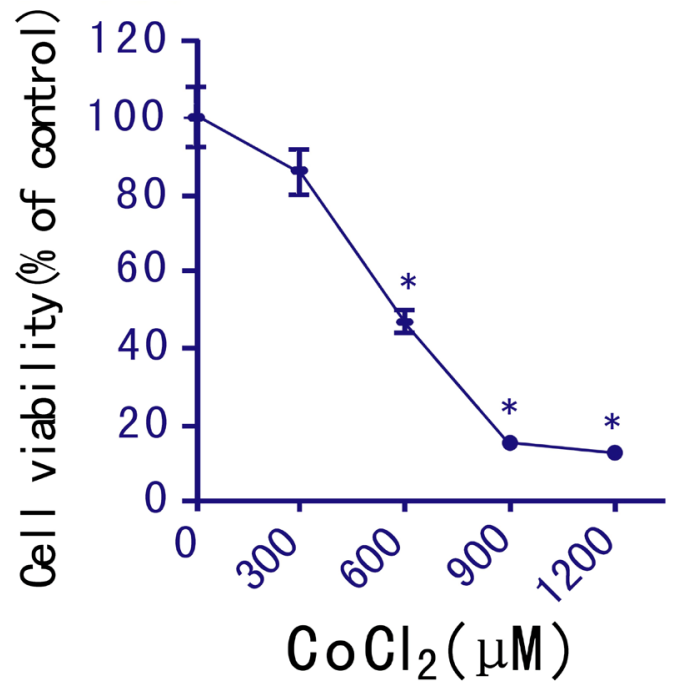

Figure 1. $\mathrm{H} 9 \mathrm{C} 2$ cells were treated with $\mathrm{CoCl}_{2}$. The concentration-dependent effects of $\mathrm{CoCl}_{2}$ on cell survival were assessed using the MTT assay. The abscissa indicates the concentration of $\mathrm{CoCl}_{2}$ and the ordinate shows the relative activity of the cell. The relative viability of cells decreased beginning at a concentration of $300 \mu \mathrm{M} \mathrm{CoCl}_{2}$ and reached the minimum at $1200 \mu \mathrm{M}$. ${ }^{\star} \mathrm{A}$ statistically significant difference compared with the control group $(P<0.05)$.

\subsection{Effects of Hypoxia on the Morphology of H9C2 Cells}

Cells in the control group were spindle-shaped, the cell membrane was smooth, grew well, displayed homogeneous adhesion, and no floating and vacuolated cells were observed (Figure 2). In the treatment group, fewer cells survived, and apoptotic cells with irregular cell membranes were observed. The number of cells displaying this phenotype increased as the $\mathrm{CoCl}_{2}$ concentration increased. After treatment with $300 \mu \mathrm{M} \mathrm{CoCl}_{2}$, the cell morphology changed, but the cell membrane was still smooth, and only a small number of cells failed to adhere and were floating in the culture bottle. At a concentration of $600 \mu \mathrm{M} \mathrm{CoCl}_{2}$, significantly fewer adherent cells were observed compared to cells treated with lower concentrations of $\mathrm{CoCl}_{2}$, and the number of floating cells increased. When the $\mathrm{CoCl}_{2}$ concentration reached $900-1200 \mu \mathrm{M}$, the number of adherent cells was further reduced, and the morphological integrity of the membrane of adherent cells was further reduced.

\subsection{Effects of Hypoxia on Oxidative Stress in H9C2 Cells}

As shown in Figure 3, after a $\mathrm{CoCl}_{2}$ treatment for $16 \mathrm{~h}$, the cells were stained with the fluorescent dye. The treatment group exhibited a significant increase in fluorescence compared with the control group. In cells treated with $0-600 \mu \mathrm{M}$ $\mathrm{CoCl}_{2}$, the fluorescence intensity gradually increased, suggesting that the level of oxidative stress increased. The highest fluorescence intensity was observed in cells treated with $600 \mu \mathrm{M} \mathrm{CoCl}_{2}$, and the value continued to increase as the concentration of the treatment increased; however, the intracellular ROS levels decreased $(P<0.05)$, suggesting that intracellular oxidative stress decreased. 
A

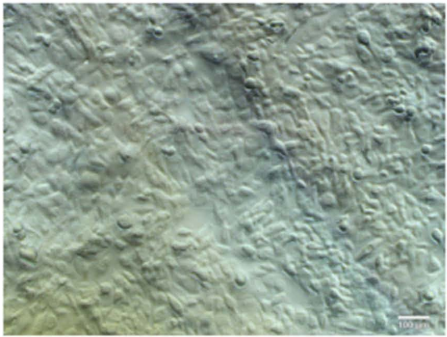

C

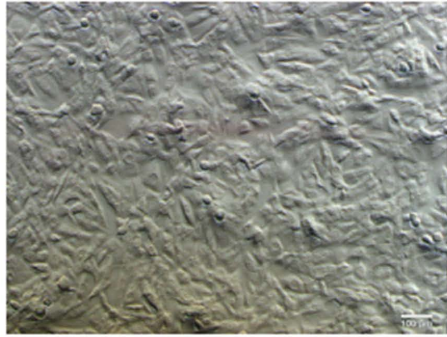

$\mathrm{E}$

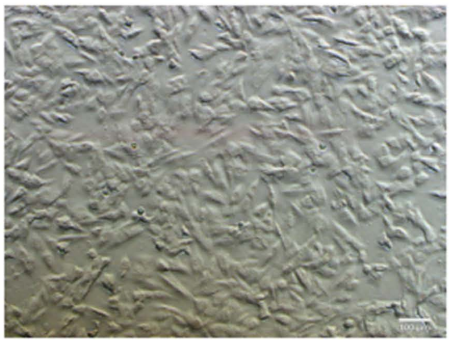

B

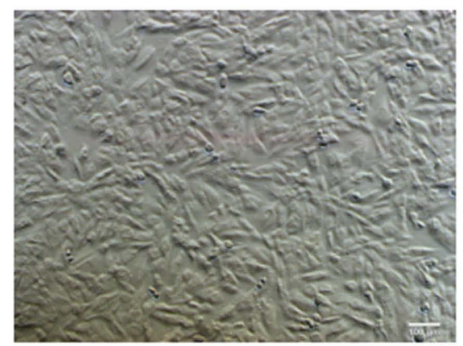

D

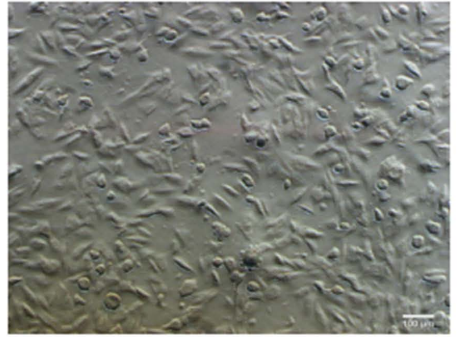

Figure 2. Cells were cultured with $\mathrm{CoCl}_{2}$ for $16 \mathrm{~h}$. A-E Cells were incubated with $\mathrm{CoCl}_{2}$ concentrations of $0,300,600,900$ or $1200 \mu \mathrm{M}$. Images of cell morphology are shown. A significant difference was not observed in cells treated with $300 \mu \mathrm{M} \mathrm{CoCl} 2$. As the concentration increased, the number of adherent cells decreased, floating cells and cell vacuolization were observed, and the cell morphology was significantly altered. Scale bar = $100 \mu \mathrm{m}$.

\subsection{Effects of $\mathrm{CoCl}_{2}$ on $\mathrm{H} 9 \mathrm{C} 2$ Cells}

The expression of HIF- $1 \alpha$ in H9C2 cells was detected by western blotting. As shown in Figure 4, after a $16 \mathrm{~h}$ treatment, a significant difference was not observed between the $300 \mu \mathrm{M}$ and control group. When the concentration of $\mathrm{CoCl}_{2}$ increased to 600,900 and $1200 \mu \mathrm{M}, \mathrm{HIF}-1 \alpha$ levels were significantly increased in a concentration-dependent manner $(P<0.05)$.

\subsection{Effects of Hypoxia on the Apoptosis of H9C2 Cells}

The expression of caspase- 3 and PARP in $\mathrm{H} 9 \mathrm{C} 2$ cells were detected by western blotting. As shown in Figure 5, significant differences in the levels of these proteins were not observed between cells treated with $300 \mu \mathrm{M} \mathrm{CoCl}_{2}$ and the control group. When the concentrations of $\mathrm{CoCl}_{2}$ in the treatment groups were 600, 900 and $1200 \mu \mathrm{M}$, caspase-3 levels were significantly increased in treated cells in a concentration-dependent manner compared with the control group $(P<0.05)$. Significant differences in PARP levels were observed in cells treated with 900 and $1200 \mu \mathrm{M} \mathrm{CoCl}_{2}(P<0.05)$. 
A

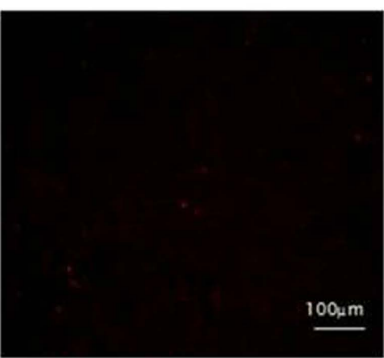

C

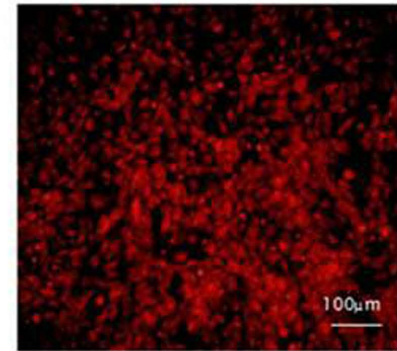

E

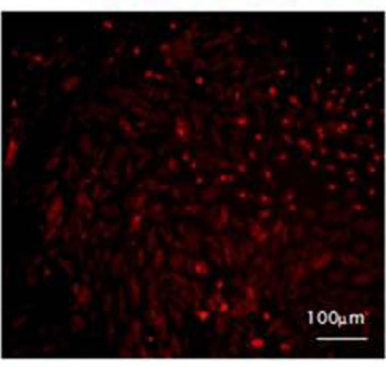

B

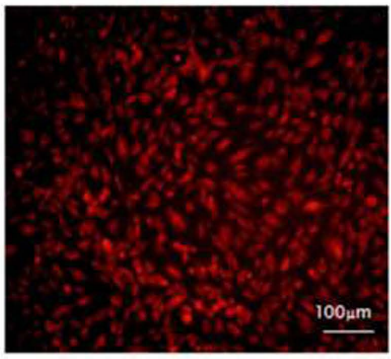

$\mathrm{D}$
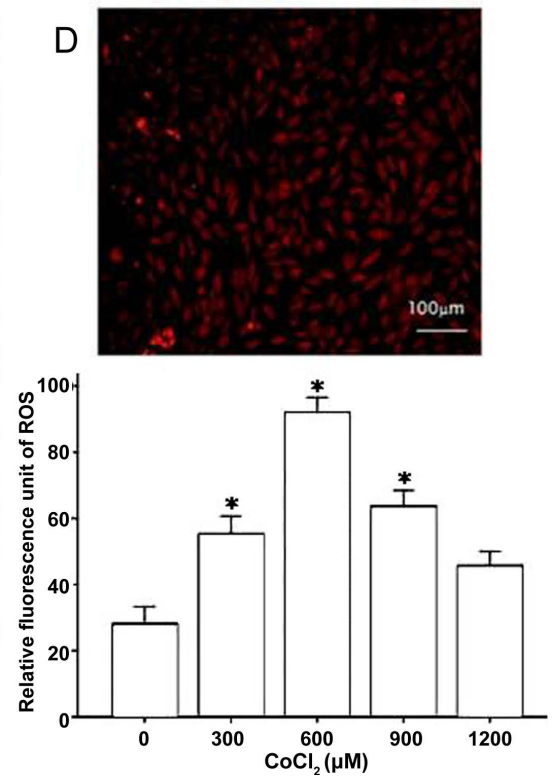

Figure 3. The intracellular ROS content was determined in cells. A-E Cells were incubated with $\mathrm{CoCl}_{2}$ concentrations of $0,300,600$, 900 or $1200 \mu \mathrm{M}$. Extremely low and almost unpredictable ROS levels were detected in the control group. As the $\mathrm{CoCl}_{2}$ concentration increased, the ROS content increased gradually, and the highest levels were observed in cells treated with $600 \mu \mathrm{M} \mathrm{CoCl}_{2}$. When the $\mathrm{CoCl}_{2}$ concentration continued to increase, the ROS content gradually decreased. ${ }^{*} \mathrm{~A}$ statistically significant difference compared with the control group $(P<0.05)$. Scale bar $=100 \mu \mathrm{m}$.

A

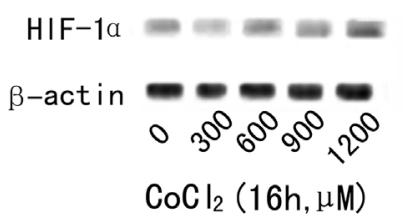

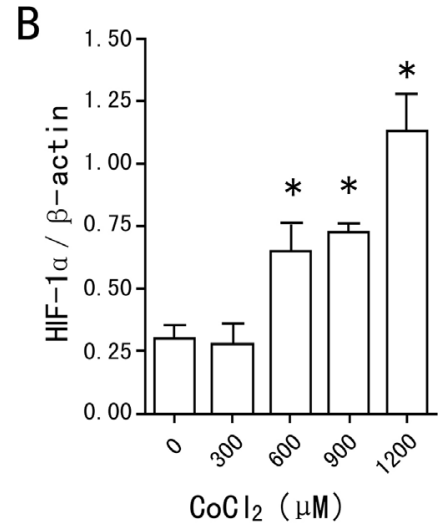

Figure 4. The degree of hypoxia was observed on $\mathrm{H} 9 \mathrm{C} 2$ cells. As the $\mathrm{CoCl}_{2}$ concentration gradually increased, the HIF- $1 \alpha$ level was not statistically significantly different between cells treated with $300 \mu \mathrm{M} \mathrm{CoCl}_{2}$ and control cells, whereas the HIF-1 $\alpha$ level was significantly increased in cells treated with $\geq 600 \mu \mathrm{M} \mathrm{CoCl} 2 .{ }^{*} \mathrm{~A}$ statistically significant difference compared with the control group $(P<0.05)$. 
A

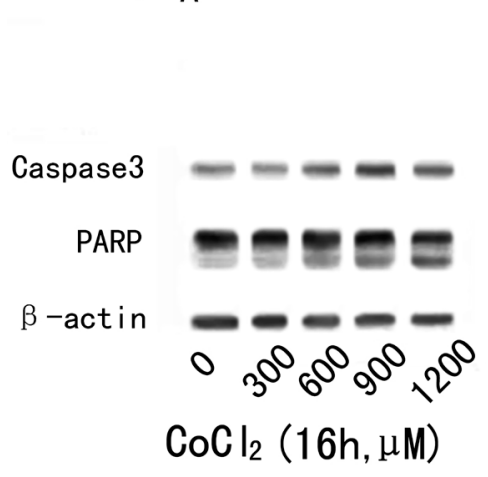

B

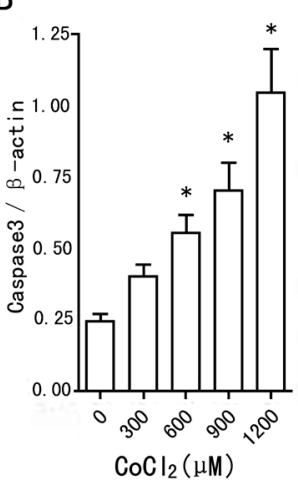

C

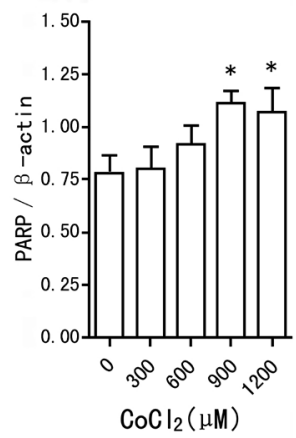

Figure 5. The levels of caspase- 3 and PARP in cells were tested. Caspase-3 levels in cells treated with $300 \mu \mathrm{M} \mathrm{CoCl} 2$ were not significantly different from control cells, but the caspase- 3 content increased in cells treated with $\geq 600 \mu \mathrm{M} \mathrm{CoCl}_{2}$. PARP levels were not significantly different between cells treated with $300 \mu \mathrm{M}$ and $600 \mu \mathrm{M} \mathrm{CoCl}_{2}$, but PARP levels increased in cells treated with $900 \mu \mathrm{M} \mathrm{CoCl}_{2}$ in a concentration-dependent manner. ${ }^{\star}$ A statistically significant difference compared with the control group $(P<0.05)$.

\subsection{Effects of Hypoxia on Autophagy in H9C2 Cells}

The levels of LC3-II and p62 in H9C2 cells were detected by western blotting. As shown in Figure 6, after a $16 \mathrm{~h}$ treatment, the levels of these proteins were not significantly different between cells treated with $300 \mu \mathrm{M} \mathrm{CoCl}$ and the control group. In cells treated with 600,900 and $1200 \mu \mathrm{M} \mathrm{CoCl}_{2}$, the LC3-II levels were significantly increased $(P<0.05)$, while the level of p62 decreased as the concentration increased, suggesting that the autophagy was induced to a statistically significant level $(P<0.05)$.

\section{Discussion}

CHD is the leading cause of cardiovascular mortality worldwide, with over 4.5 million deaths occurring in the developing world [23]. CHD is a multifactorial heritable disease caused by a variety of risk factors related to the oxygen and energy needs of the myocardial tissue [24]. However, in patients with CHD, the exact relationships among oxidative stress, autophagy and apoptosis have not been confirmed. Moreover, researchers have not yet determined whether other pathological processes occur in response to hypoxia. To date, few reports have examined the relationships among these three processes. We believe that a better understanding of these relationships will facilitate the prevention and treatment of $\mathrm{CHD}$. In our study, we treated $\mathrm{H} 9 \mathrm{C} 2$ cells with $\mathrm{CoCl}_{2}$ to mimic cardiomyocyte hypoxia. Hypoxia affected cell viability and growth, based on the results from the MTT assay and microscopic observations. Hypoxic cells also exhibited increased HIF- $1 \alpha$ and ROS levels, confirming that hypoxia induced oxidative stress. Additionally, caspase-3 and PARP levels were increased, indicating that hypoxia induced apoptosis. Simultaneously, intracellular LC3-II levels increased and p62 levels decreased, confirming that hypoxia induced autophagy in hypoxic cells. Based on the results of these experiments, hypoxia induced oxidative stress, 

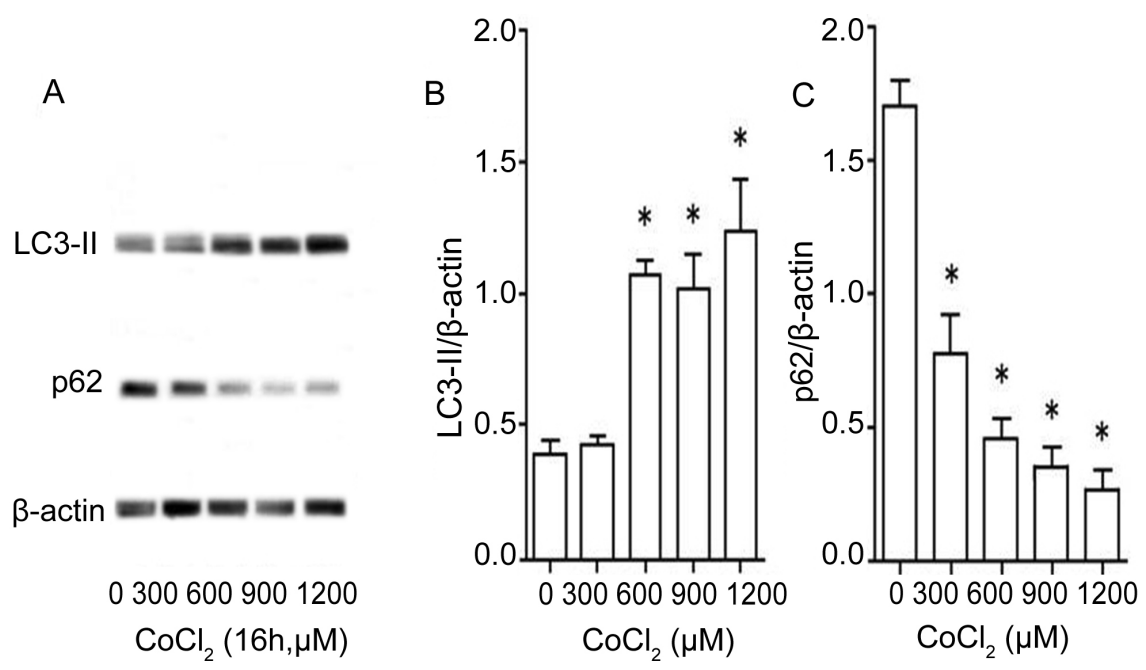

Figure 6. Levels of LC3-II and p62 in cells treated with $\mathrm{CoCl}_{2}$ for 16 h. Significant differences in LC3-II levels were not observed between cells treated with $300 \mu \mathrm{M} \mathrm{CoCl}_{2}$ and the control group. LC3-II levels increased in cells treated with $\geq 600 \mu \mathrm{M} \mathrm{CoCl}_{2}$ in a concentration-dependent manner, whereas the p62 levels gradually decreased. ${ }^{\star} \mathrm{A}$ statistically significant difference compared with the control group $(P<0.05)$.

apoptosis and autophagy in $\mathrm{H} 9 \mathrm{C} 2$ cells. Based on these results, hypoxia induces oxidative stress, apoptosis and autophagy in cardiomyocytes. Autophagy is a double-edged sword. At a low level, autophagy can resist oxidative stress and protect cardiomyocytes from oxidative stress, while high level autophagy can promote apoptosis of cardiomyocytes.

Hypoxia inhibits HIF- $1 \alpha$ hydroxylation, thereby promoting its accumulation; hypoxia also allows the mitochondria to release more ROS into the cytosol [25]. In our study, both ROS and HIF- $1 \alpha$ levels were increased in the treatment group, indicating that we successfully induced oxidative stress in hypoxic cells using $\mathrm{CoCl}_{2}$. Increased ROS production leads to DNA, protein and lipid modifications, and activates stress-signaling pathways leading to heart failure in subjects with I/R injury [26]. Several studies have reported the occurrence of both autophagy and oxidative stress in response to cell death stimuli. However, very little experimental evidence for a direct role for autophagic cell death in ROS/RNSor oxidative stress-mediated toxicity has been reported [5]. For example, oxidative stress was reported to induce cell death via both autophagy and necrosis, and knockdown of Atg7 (Atg7 is a core autophagy protein [27]) only delayed early cell death progression in primary murine cortical neurons [28]. In contrast, a number of recent studies have reported a protective effect of autophagy on oxidative stress-induced cell death [5]. For example, lipocalin-2 (NGAL) attenuates autophagy to exacerbate cardiac apoptosis induced by myocardial ischemia [29]. Oxidative stress is associated with increased formation of ROS that contribute to the pathophysiology of I/R injury. Myocardial I/R injury must be prevented to achieve a successful CHD surgery and recovery. Novel treatments that inhibit or ameliorate the disease process must be developed to allow sufficient time for subsequent treatment. 
Autophagy acts as a survival mechanism under stress conditions, maintaining the cellular integrity by regenerating metabolic precursors and clearing subcellular debris [27]. This process contributes to basal cellular and tissue homeostasis, regulates development in higher organisms, and can affect pathogenesis [27]. During autophagy, autophagosomes fuse with lysosomes to form autolysosomes, and the autophagosome content is degraded by lysosomal hydrolases. LC3 in the autolysosome lumen is simultaneously degraded [30]. Therefore, an increase in LC3 levels may result from a reduced turnover of autophagosomes rather than from increased autophagy activity, and p62 is known to be degraded together with the autophagosome content [30]. In our study, LC3-II levels were increased concomitantly with decreased p62 levels, indicating that autophagy was induced by the $\mathrm{CoCl}_{2}$ treatment in a concentration-dependent manner, consistent with other studies [31]. Autophagy may exhibit dual functions in hypoxia-induced cell damage. In most cases, the activation of autophagy protects the cell; however, excessive activation of autophagy may also mediate cell death through a process known as autophagic cell death (ACD) [32]. Nevertheless, the activation of autophagy has also been reported to induce apoptosis, and some proteins that play important roles in autophagy may also induce the apoptosis signal transduction pathway [32].

Apoptosis, a form of programmed cell death, occurs in a wide range of physiological and pathological situations. It is characterized by cell shrinkage, programmed DNA degradation, and increased expression of caspase-3 [33]. In our study, the expression of caspase- 3 increased as the $\mathrm{CoCl}_{2}$ concentration increased, suggesting that the degree of hypoxia was positively correlated with the level of apoptosis. However, a limitation of cleaved caspase- 3 was not detected to confirm that hypoxia induced the activation of caspase-3. PARP is a critical enzyme involved in the repair of DNA strand breaks [34]. In the present study, PARP levels increased in the presence of higher concentrations of $\mathrm{CoCl}_{2}$. Oxidative stress-induced production of ROS likely damages the DNA and increases intracellular PARP expression. Excess ROS also induce the oxidative modification of cellular macromolecules, inhibit protein function, and induce cell death [35]. In our experiment, the highest ROS levels were observed in cells treated with the IC50 concentration of $\mathrm{CoCl}_{2}$, and the ROS level decreased at higher concentrations, which may be related to the increased levels of apoptosis and autophagy, which reduced ROS production. The relationship between autophagy and apoptosis is complex, as they share the same set of cellular regulatory proteins and are closely linked. Autophagy has been reported to inhibit or delay the occurrence of apoptosis, as well as to promote apoptosis [19]. In fact, the role of autophagy itself is two-way, that is, to protect or promote the damage depending on experiments, as shown in the present study, excess autophagy likely promoted the apoptosis of cardiomyocytes and this is only based on results for marker proteins of autophagy and apoptosis, while the actual occurrence of autophagy and apoptosis requires confirmation using additional experiments. 
In our study, cell hypoxia was simulated by the $\mathrm{CoCl}_{2}$ treatment, and cells cultured under hypoxic conditions exhibited oxidative stress, autophagy and apoptosis. As the $\mathrm{CoCl}_{2}$ concentration increased, the levels of autophagy and apoptosis gradually increased, suggesting that both processes may employ a common molecular mechanism or signaling pathway. Moreover, when the concentration of $\mathrm{CoCl}_{2}$ was less than $600 \mu \mathrm{M}$, the ROS level increased as the $\mathrm{CoCl}_{2}$ concentration increased, but a significant difference in the increase in caspase- 3 levels was not observed. Thus, we speculated that intracellular antioxidants such as glutathione might block ROS-induced apoptosis, and the cells are still in the negative feedback regulation stage. However, when the concentration of $\mathrm{CoCl}_{2}$ exceeded $600 \mu \mathrm{M}$, the level of apoptosis is increased, but the ROS content was not increased. Therefore, treatment with high doses $\mathrm{CoCl}_{2}$ likely influenced the intracellular sources from which ROS were produced through effects on the mitochondrial integrity and electron transfer chain, and may have even directly caused cell death to decrease the ROS content. Based on these findings, oxidative stress may not be the most important factor contributing to apoptosis. We also noticed that at $\mathrm{CoCl}_{2}$ concentrations less than $600 \mu \mathrm{M}$, oxidative stress occurred, but the LC3-II level did not increase, potentially due to a cellular reduction mechanism that blocks the transduction of ROS signals. When the concentration exceeded $600 \mu \mathrm{M}$, the ROS content decrease and the level of autophagy increased. We speculated that autophagy may function as an antioxidant and increase the phagocytosis of oxidative stress-related oxidases and organelles, such as NADPH oxidase, mitochondria and other structures, in vivo to decrease the ROS content decreased. In this experiment, when the concentration of $\mathrm{CoCl}_{2}$ was less than $600 \mu \mathrm{M}$, the levels of autophagy- and apoptosis-related proteins were not increased, and when the concentration of $\mathrm{CoCl}_{2}$ exceeded $600 \mu \mathrm{M}$, the levels of these proteins increased. We also confirmed that $\mathrm{CoCl}_{2}$ mimicked cardiomyocyte hypoxia by inhibiting cell growth, and inducing oxidative stress, apoptosis and autophagy. Moreover, when the concentration of $\mathrm{CoCl}_{2}$ was 600 $\mu \mathrm{M}$, the maximum level of intracellular oxidative stress was observed. As the level of hypoxia increased, cells may have alleviated intracellular oxidative stress by increasing autophagy; however, when the concentration of $\mathrm{CoCl}_{2}$ continued to increase, cells were more likely to undergo apoptosis following autophagy than after oxidative stress in our opinion. Hypoxia-induced oxidative stress leads to irreversible damage in many diseases characterized by ischemia. Treatments that decrease ROS production or increase the ROS scavenging ability of the system may exert beneficial effects on the disease. Endogenous mechanism or exogenous intervention can coordinate the function of autophagy and inhibit the damage of oxidative stress and apoptosis to myocardium.

The relationship among oxidative stress, autophagy and apoptosis is complex, and mutual influence is achieved through multiple signaling systems. Further researches are needed to verify the changes in cellular and molecular signals of oxidative stress, autophagy and apoptosis in the heart. 


\section{Acknowledgements}

This work was supported by grants from the National Natural Sciences Foundation of China (81200150 and 81660064), Hainan Province Natural Science Foundation (20158338), Hainan Provincial Foundation for Health Department (14A210227), Cultivating Fund of Hainan Medical University (HY2014-007), Student's Innovative Project of Hainan Medical College (HYCX2015003), and National Students' Innovation and Entrepreneurship Training Program (201611810052).

\section{Conflicts of Interest}

The authors declare no conflict of interest.

\section{References}

[1] Levine, B.D. (2015) Going High with Heart Disease: The Effect of High Altitude Exposure in Older Individuals and Patients with Coronary Artery Disease. High Altitude Medicine \& Biology, 16, 89-96. https://doi.org/10.1089/ham.2015.0043

[2] Yu, H.H., Xu, Q., Chen, H.P., Wang, S., Huang, X.S., Huang, Q.R., et al. (2013) Stable Overexpression of DJ-1 Protects H9C2 Cells against Oxidative Stress under a Hypoxia Condition. Cell Biochemistry and Function, 31, 643-651. https://doi.org/10.1002/cbf.2949

[3] Liu, B., Che, W., Xue, J., Zheng, C., Tang, K., Zhang, J., et al. (2013) SIRT4 Prevents Hypoxia-Induced Apoptosis in H9C2 Cardiomyoblast Cells. Cellular Physiology and Biochemistry, 32, 655-662. https://doi.org/10.1159/000354469

[4] Gui, L., Liu, B. and Lv, G. (2016) Hypoxia Induces Autophagy in Cardiomyocytes via a Hypoxia-Inducible Factor 1-Dependent Mechanism. Experimental and Therapeutic Medicine, 11, 2233-2239. https://doi.org/10.3892/etm.2016.3190

[5] Navarro-Yepes, J., Burns, M., Anandhan, A., Khalimonchuk, O., del Razo, L.M., Quintanilla-Vega, B., et al. (2014) Oxidative Stress, Redox Signaling, and Autophagy: Cell Death versus Survival. Antioxidants and Redox Signaling, 21, 66-85. https://doi.org/10.1089/ars.2014.5837

[6] Rupérez, A.I., Gil, A. and Aguilera, C.M. (2014) Genetics of Oxidative Stress in Obesity. International Journal of Molecular Sciences, 15, 3118-3144. https://doi.org/10.3390/ijms15023118

[7] Yu, B., Meng, F., Yang, Y., Liu, D. and Shi, K. (2016) NOX2 Antisense Attenuates Hypoxia-Induced Oxidative Stress and Apoptosis in Cardiomyocyte. International Journal of Molecular Sciences, 13, 646-652. https://doi.org/10.7150/ijms.15177

[8] Li, S.Y., Fu, Z.J. and Lo, A.C. (2012) Hypoxia-Induced Oxidative Stress in Ischemic Retinopathy. Oxidative Medicine and Cellular Longevity, 2012, Article ID: 426769. https://doi.org/10.1155/2012/426769

[9] Wang, X., Wu, D., Yang, L., Gan, L. and Cederbaum, A.I. (2013) Cytochrome P450 2E1 Potentiates Ethanol Induction of Hypoxia and HIF-1 $\alpha$ in Vivo. Free Radical Biology \& Medicine, 63, 175-186. https://doi.org/10.1016/j.freeradbiomed.2013.05.009

[10] Li, D.L. and Hill, J.A. (2014) Cardiomyocyte Autophagy and Cancer Chemotherapy. Journal of Molecular and Cellular Cardiology, 71, 54-61. https://doi.org/10.1016/j.yjmcc.2013.11.007

[11] Zhang, Z., Singh, R. and Aschner, M. (2016) Methods for the Detection of Auto- 
phagy in Mammalian Cells. Current Protocols in Toxicology, 69, 20.12.1-20.12.26.

[12] Bjørkøy, G., Lamark, T., Pankiv, S., Øvervatn, A., Brech, A. and Johansen, T. (2009) Monitoring Autophagic Degradation of p62/SQSTM1. Methods in Enzymology, 452, 181-197. https://doi.org/10.1016/S0076-6879(08)03612-4

[13] Qi, D. and Fu, M. (2012) Cardiomyocyte Apoptosis in Heart Development: Methods and Protocols. Methods in Molecular Biology, 843, 191-197. https://doi.org/10.1007/978-1-61779-523-7 18

[14] Nestal de Moraes, G., Carvalho, É., Maia, R.C. and Sternberg, C. (2011) Immunodetection of Caspase-3 by Western Blot Using Glutaraldehyde. Analytical Biochemistry, 415, 203-205. https://doi.org/10.1016/j.ab.2011.04.032

[15] Aslan Koşar, P., Tuncer, H., Cihangir Uğuz, A., Espino Palma, J., Darıcı, H., Onaran, İ., et al. (2015) The Efficiency of Poly(ADP-Ribose) Polymerase (PARP) Cleavage on Detection of Apoptosis in an Experimental Model of Testicular Torsion. International Journal of Experimental Pathology, 96, 294-300. https://doi.org/10.1111/iep.12137

[16] Chen, J.R., Wei, J., Wang, L.Y., Zhu, Y., Li, L., Olunga, M.A., et al. (2015) Cardioprotection against Ischemia/Reperfusion Injury by QiShenYiQi Pill ${ }^{\oplus}$ via Ameliorate of Multiple Mitochondrial Dysfunctions. Drug Design, Development and Therapy, 15, 3051-3066. https://doi.org/10.2147/DDDT.S82146

[17] Zhong, X., Li, X., Qian, L., Xu, Y., Lu, Y., Zhang, J., et al. (2012) Glycine Attenuates Myocardial Ischemia-Reperfusion Injury by Inhibiting Myocardial Apoptosis in Rats. Journal of Biomedical Research, 26, 346-354. https://doi.org/10.7555/JBR.26.20110124

[18] Chen, J.W., Ni, B.B., Li, B., Yang, Y.H., Jiang, S.D. and Jiang, L.S. (2014) The Responses of Autophagy and Apoptosis to Oxidative Stress in Nucleus Pulposus Cells: Implications for Disc Degeneration. Cellular Physiology and Biochemistry, 34, 1175-1189. https://doi.org/10.1159/000366330

[19] Tong, X.X., Wu, D., Wang, X., Chen, H.L., Chen, J.X., Wang, X.X., et al. (2012) Ghrelin Protects against Cobalt Chloride-Induced Hypoxic Injury in Cardiac H9C2 Cells by Inhibiting Oxidative Stress and Inducing Autophagy. Peptides, 38, 217-227. https://doi.org/10.1016/j.peptides.2012.06.020

[20] Ouyang, F., Huang, H., Zhang, M., Chen, M., Huang, H., Huang, F., et al. (2016) HMGB1 Induces Apoptosis and EMT in Association with Increased Autophagy Following H/R Injury in Cardiomyocytes. International Journal of Molecular Medicine, 37, 679-689. https://doi.org/10.3892/ijmm.2016.2474

[21] Mao, S.Y., Meng, X.Y., Xu, Z.W., Zhang, W.C., Jin, X.H., Chen, X., et al. (2017) The Role of ZFP580, a Novel Zinc Finger Protein, in TGF-Mediated Cytoprotection against Chemical Hypoxia Induced Apoptosis in H9C2 Cardiac Myocytes. Molecular Medicine Reports, 15, 2154-2162. https://doi.org/10.3892/mmr.2017.6236

[22] Rashidlamir, A., Attarzadeh Hosseini, S.R., Hejazi, K. and Motevalli Anberani, S.M. (2016) The Effect of Eight Weeks Resistance and Aerobic Training on Myostatin and Follistatin Expression in Cardiac Muscle of Rats. Journal of Cardiovascular and Thoracic Research, 8, 164-169. https://doi.org/10.15171/jcvtr.2016.33

[23] Okrainec, K., Banerjee, D.K. and Eisenberg, M.J. (2004) Coronary Artery Disease in the Developing World. American Heart Journal, 148, 7-15. https://doi.org/10.1016/j.ahj.2003.11.027

[24] Liu, Q., Liang, Y., Zou, P., Ni, W.X., Li, Y.G. and Chen, S.M. (2013) Hypoxia-Inducible Factor-1 $\alpha$ Polymorphisms Link to Coronary Artery Collateral Development and Clinical Presentation of Coronary Artery Disease. Biomedical Papers of 
the Medical Faculty of Palacký University, Olomouc, Czech Republic, 157, 340-345. https://doi.org/10.5507/bp.2013.061

[25] Guzy, R.D., Hoyos, B., Robin, E., Chen, H., Liu, L., Mansfield, K.D., et al. (2005) Mitochondrial Complex III Is Required for Hypoxia-Induced ROS Production and Cellular Oxygen Sensing. Cell Metabolism, 1, 401-408. https://doi.org/10.1016/j.cmet.2005.05.001

[26] Sengupta, A., Molkentin, J.D., Paik, J.H., Chen, H., Liu, L., Mansfield, K.D., et al. (2011) FoxO Transcription Factors Promote Cardiomyocyte Survival upon Induction of Oxidative Stress. The Journal of Biological Chemistry, 286, 7468-7478. https://doi.org/10.1074/jbc.M110.179242

[27] Choi, A.M., Ryter, S.W. and Levine, B. (2013) Autophagy in Human Health and Disease. New England Journal of Medicine, 368, 1845-1846.

https://doi.org/10.1056/NEJMra1205406

[28] Higgins, G.C., Devenish, R.J., Beart, P.M. and Nagley, P. (2012) Transitory Phases of Autophagic Death and Programmed Necrosis during Superoxide-Induced Neuronal Cell Death. Free Radical Biology \& Medicine, 53, 1960-1967. https://doi.org/10.1016/j.freeradbiomed.2012.08.586

[29] Sung, H.K., Chan, Y.K., Han, M., Jahng, J.W.S., Song, E., Danielson, E., et al. (2017) Lipocalin-2 (NGAL) Attenuates Autophagy to Exacerbate Cardiac Apoptosis Induced by Myocardial Ischemia. Journal of Cellular Physiology, 232, 2125-2134. https://doi.org/10.1002/jcp.25672

[30] Masschelein, E., Van Thienen, R., D’Hulst, G., Hespel, P., Thomis, M. and Deldicque, L. (2014) Acute Environmental Hypoxia Induces LC3 Lipidation in a Genotype-Dependent Manner. FASEB Journal, 28, 1022-1034. https://doi.org/10.1096/fj.13-239863

[31] Chen, R., Jiang, T., She, Y., Xu, J., Li, C., Zhou, S., et al. (2017) Effects of Cobalt Chloride, a Hypoxia-Mimetic Agent, on Autophagy and Atrophy in Skeletal C2C12 Myotubes. BioMed Research International, 2017, Article ID: 7097580. https://doi.org/10.1155/2017/7097580

[32] Li, M., Tan, J., Miao, Y., Lei, P. and Zhang, Q. (2015) The Dual Role of Autophagy under Hypoxia-Involvement of Interaction between Autophagy and Apoptosis. Apoptosis, 20, 769-777. https://doi.org/10.1007/s10495-015-1110-8

[33] Fan, G., Yu, J., Asare, P.F., Wang, L., Zhang, H., Zhang, B., et al. (2016) Danshensu Alleviates Cardiac Ischaemia/Reperfusion Injury by Inhibiting Autophagy and Apoptosis via Activation of mTOR Signalling. Journal of Cellular and Molecular Medicine, 20, 1908-1919. https://doi.org/10.1111/jcmm.12883

[34] Maciag, A.E., Holland, R.J., Kim, Y., Kumari, V., Luthers, C.E., Sehareen, W.S., et al. (2014) Nitric Oxide (NO) Releasing Poly ADP-Ribose Polymerase 1 (PARP-1) Inhibitors Targeted to Glutathione S-Transferase P1-Over Expressing Cancer Cells. Journal of Medicinal Chemistry, 57, 2292-2302. https://doi.org/10.1021/jm401550d

[35] Circu, M.L. and Aw, T.Y. (2010) Reactive Oxygen Species, Cellular Redox Systems, and Apoptosis. Free Radical Biology \& Medicine, 48, 749-762.

https://doi.org/10.1016/j.freeradbiomed.2009.12.022 